\title{
Ionic Additives to Increase Electrochemical Utilization of Sulfur Cathode for Li-S Batteries
}

\author{
Min Ji Seong and Taeeun Yim* \\ Department ofChemistry, Research Institute of Basic Sciences, College of Natural Science, Incheon National University, 119, \\ Academy-ro, Yeonsu-gu, Incheon, 22012, Korea
}

\begin{abstract}
The high theoretical specific capacity of lithium-sulfur (Li-S) batteries makes them a more promising energy storage system than conventional lithium-ion batteries (LIBs). However, the slow kinetics of the electrochemical conversion reaction seriously hinders the utilization of $\mathrm{Li}-\mathrm{S}$ as an active battery material and has prevented the successful application of Li-S cells. Therefore, exploration of alternatives that can overcome the sluggish electrochemical reaction is necessary to increase the performance of Li-S batteries. In this work, an ionic liquid (IL) is proposed as a functional additive to promote the electrochemical reactivity of the Li-S cell. The sluggish electrochemical reaction is mainly caused by precipitation of low-order polysulfide (l-PS) onto the positive electrode, so the IL is adopted as a solubilizer to remove the precipitated $l$-PS from the positive electrode to promote additional electron transfer reactions. The ILs effectively dissolve $l$-PS and greatly improve the electrochemical performance by allowing greater utilization of $l$-PS, which results in a higher initial specific capacity, together with a moderate retention rate. The results presented here confirmed that the use of an IL as an additive is quite effective at enhancing the overall performance of the Li-S cell and this understanding will enable the construction of highly efficient Li-S batteries.
\end{abstract}

Keywords : Lithium Sulfur Batteries, Electrolyte, Polysulfide, Additive

Received : 5 October 2020, Accepted : 26 January 2021

\section{Introduction}

The high theoretical specific capacity (1672 mA h $\mathrm{g}^{-1}$ ) of the lithium-sulfur (Li-S) batteries makes them a promising energy storage system for future applications, such as plug-in-hybrid electric vehicles (PHEVs), electric vehicles (EVs), and energy storage systems (ESSs) [1-5]. Nevertheless, several challenging issues still hinder the successful commercialization of Li-S batteries. One significant problem that has plagued the commercialization of Li-S batteries is the continuous and rapid capacity fading of the cell, which arises due to the unique characteristics of polysulfide (PS) [6-10]. High-order polysulfide ( $h$-PS, $\mathrm{Li}_{2} \mathrm{~S}_{\mathrm{x}}, 4 \leq \mathrm{x} \leq 8$ ) is quite soluble in electrolyte, so it easily passivates on the lithium metal (Li) anode through a chemical conversion reac-

*E-mail address: yte0102@inu.ac.kr

DOI: https://doi.org/10.33961/jecst.2020.01494

This is an open-access article distributed under the terms of the Creative Commons Attribution Non-Commercial License (http://creativecommons.org/licenses/by-nc/4.0) Attribution Non-Commercial License (http://creativecommons.org/licenses/by-nc/4.0)
which permits unrestricted non-commercial use, distribution, and reproduction in any which permits unrestricted non-commercial use, distri
medium, provided the original work is properly cited. tion of $h$-PS to $\mathrm{Li}_{2} \mathrm{~S}$ and this causes serious amounts of active material loss. This issue has led to the generation of several kinds of recipes for designating restriction spaces that prevent PS diffusion, such as encapsulation of the sulfur cathode, modifying the surface properties with a hydrophilic layer, using a metal oxide additive as a PS reservoir, and construction of a dual-functionalized cathode architecture, along with the addition of lithium nitrate $\left(\mathrm{LiNO}_{3}\right)$ in the electrolyte [11-15]. The end result is the formation of a protective layer on the Li metal anode that suppresses the chemical consumption of PS, as well as minimizing PS diffusion.

Another serious obstacle is the slow kinetics of the electrochemical conversion reaction of PS [16-18]. Because the low-order polysulfide ( $l$-PS, $\mathrm{Li}_{2} \mathrm{~S}_{\mathrm{x}}, 1 \leq \mathrm{x}$ $\leq 2)$ is insoluble in the electrolyte, the electrochemical conversion of $h$-PS gives a discharge product on cathode surface in the form of precipitated $l$-PS. This means that the electrochemically active sites (which are responsible for the electrochemical reaction) gradually disappear with the increasing insulation by 


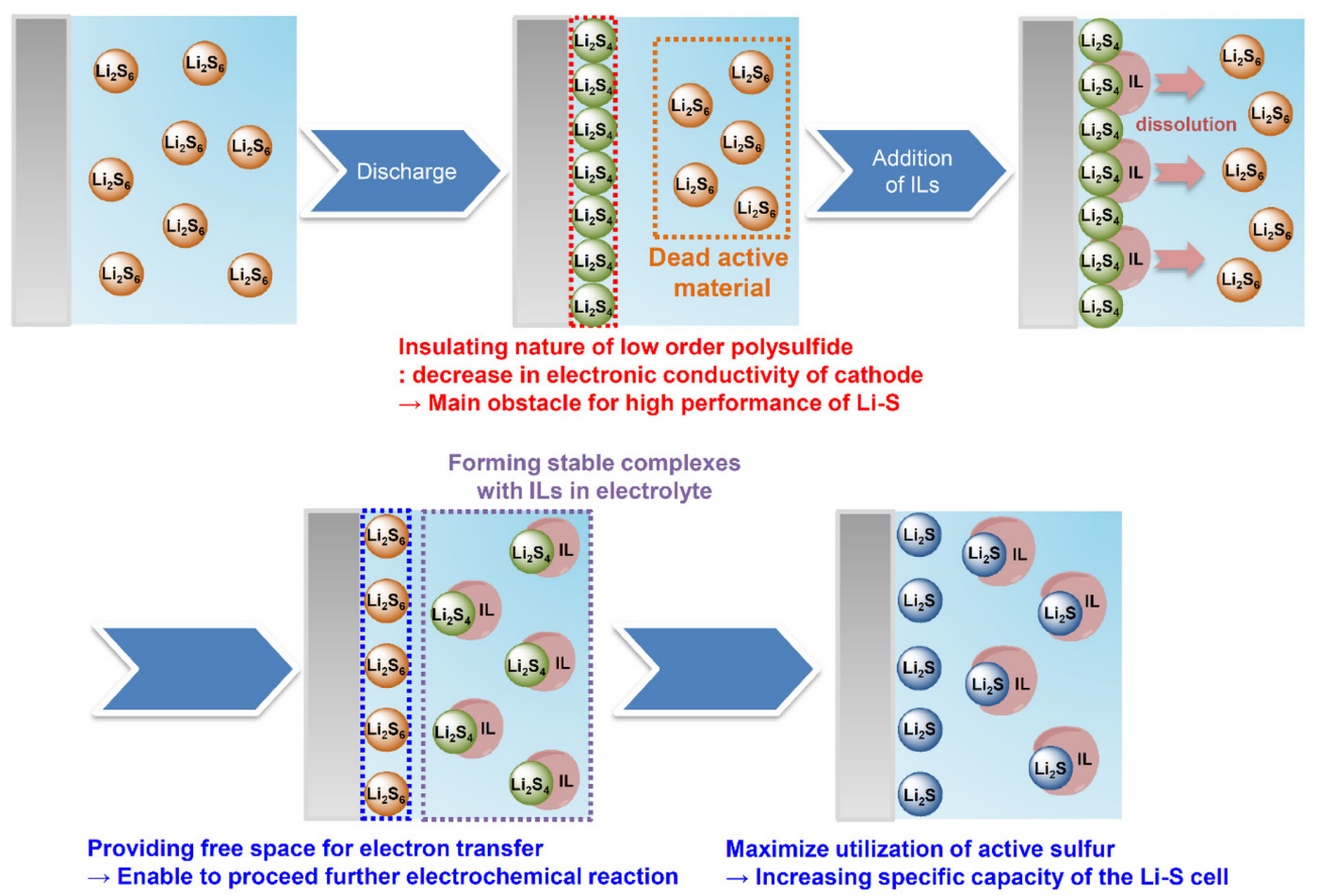

Fig. 1. The mechanism to forming stable complexes of $l$-PS and ILs.

accumulating $l$-PS, which disrupts any additional utilization of the remaining active material in the electrolyte [19-21]. As a result, the insoluble nature of $l$ PS considerably affects the electrochemical performance including the cycle performance and the rate capability of the Li-S cell. Therefore, exploration for an effective approach to enhance the sluggish electrochemical conversion of PS is essential for achieving high performance of Li-S batteries.

In this work, we report the use of a task-specific ionic liquid (IL)-based additive that accelerates the slow electrochemical reaction of PS in Li-S batteries. During the past two decades, ILs have been highlighted as alternative solvents to replace conventional electrolytes because of their unique physicochemical properties [22-24]. The most noticeable characteristic of the ILs is their amphiphilic nature - an IL is able to dissolve many kinds of solutes, regardless of whether they are organic or inorganic species because the IL is composed of an organic cation and an inorganic anion. This means that the IL can be utilized to enhance the sluggish electrochemical conversion of the Li-S cell as even if the less soluble $l$-PS is deposited on the cathode surface, the $l$-PS is still able to stay in the electrolyte by the formation of stable complexes with the IL, thereby freeing up space to facilitate further electron transfer reactions on the cathode surface (Fig. 1). Therefore, the overall electrochemical performance of the Li-S cell is expected to improve through control of the interfacial chemistry between the cathode and the electrolyte. Several attempts have been made to use an IL as an additive [25-27]; however, none of the previous studies clearly demonstrated a role for the IL in the electrochemical performance of the Li-S cell, even though the work provides informative clues about how to design more efficient Li-S batteries. With these considerations in mind, systematic studies are performed in the present study to probe the effect of ILs on the electrochemical performance of the Li-S cell.

\section{Experimental section}

To a stirred solution of 1-methylpyrrolidine (50.0 mmol, Aldrich) in acetonitrile $(100 \mathrm{~mL})$ was added butyl halide (55.0 mmol, Aldrich) dropwise at 
$0^{\circ} \mathrm{C}$, and the reaction mixture was stirred for $24 \mathrm{~h}$ at room temperature. After the reaction was completed, the solvent was removed under reduced pressure and the crude 1-butyl-1-methylpyrrolidnium halide was washed twice with ethyl acetate $(20 \mathrm{~mL})$ to remove residual impurities.

The electrochemical windows of the ILs were examined by linear sweep voltammetry (LSV) with an electrochemical workstation (SP-300, Biologic). The standard electrolyte (TEGDME:DME:DOL = 1:1:1 with 1.0 M LiTFSI) was provided by PanaxEtec and additional 0.1 M ILs were added to the standard electrolyte to monitor the electrochemical behavior of the ILs. For the LSV measurement, a glassy carbon electrode was used as the working electrode $\left(7.07 \times 10^{-2} \mathrm{~cm}^{2}\right)$ and lithium foil was used for the counter and reference electrodes. Scanning was conducted from 3.0 to $4.2 \mathrm{~V}$ (vs. $\mathrm{Li} / \mathrm{Li}^{+}$) and 3.0 to $0.0 \mathrm{~V}$ (vs. $\mathrm{Li} / \mathrm{Li}^{+}$) at a scan rate of $0.1 \mathrm{mV} \mathrm{s}^{-1}$.

The solubility of the ILs was verified by performing storage tests. To an electrolyte solution (TEGDME:DME:DOL=1:1:1 with 1.0 M LiTFSI + $0.1 M$ ILs), additional $0.01 M \mathrm{Li}_{2} \mathrm{~S}$ (Aldrich) was added under an argon (Ar) atmosphere and the mixture was stirred for three days. After completion of the storage test, supernatants from each sample were collected and their electrochemical behaviors were examined by cyclic voltammetry $(\mathrm{CV})$ to confirm $\mathrm{Li}_{2} \mathrm{~S}$ dissolution in the electrolyte. For CV measurements, a glassy carbon electrode was used as working electrode $\left(7.07 \times 10^{-2} \mathrm{~cm}^{2}\right)$ and lithium foil was used for the counter and reference electrodes. Scanning was conducted in the ranged from 3.0 to $1.5 \mathrm{~V}$ (vs. $\left.\mathrm{Li} / \mathrm{Li}^{+}\right)$at a scan rate of $0.1 \mathrm{mV} \mathrm{s}^{-1}$.

The IL performance in the Li-S cell was evaluated using a positive electrode prepared as follows. Elemental sulfur (325 mesh, Alfa Aesar) was mixed with poly(ethylene oxide) (PEO, MW: 600,000, Aldrich) and carbon black (EC 600 JD, Ketjen Black) in acetonitrile (MeCN, anhydrous, Aldrich) at a ratio of 50:25:25 wt.\%. After homogenization, the slurry was coated onto a $26 \mu \mathrm{m}$ thick carbon-coated aluminum current collector (Purichem) with a scalpel. The resulting cathode was dried at $60^{\circ} \mathrm{C}$ for $24 \mathrm{~h}$, cut into $\varnothing 12 \mathrm{~mm}$ disks, and dried for $24 \mathrm{~h}$ under vacuum at room temperature. The electrode loading was approximately $3.50 \pm 0.15 \mathrm{mg} \mathrm{cm}^{-2}$.

The 2032 coin-type cells were assembled with a $\varnothing 12$ mm-diameter electrode, lithium foil, a PE sep- arator (Asahi), and electrolyte (TEGDME:DME:DOL = 1:1:1 with 1.0 M LiTFSI + 0.1 M ILs). The galvanostatic discharging-charging tests were performed with a Toscat-3100 in the potential range of $2.8-1.5 \mathrm{~V}$ (vs. $\mathrm{Li} / \mathrm{Li}^{+}$) at a $2.0 \mathrm{C}$ rate at $25^{\circ} \mathrm{C}$. Upon completion of the galvanostatic cyclings, the cells were dismantled in an Ar-filled glove box with $<0.5 \mathrm{ppm}$ of $\mathrm{H}_{2} \mathrm{O}$ and $<1 \mathrm{ppm}$ of $\mathrm{O}_{2}$ and the positive electrode was washed with fresh 1,2-dimethoxyethane (DME, Aldrich). The surfaces were analyzed by field-emission-scanning electron microscopy (FE-SEM, Thermo keytex).

\section{Results and Discussions}

Because the usual operating potential of the Li-S cell is in the range of $1.5-3.0 \mathrm{~V}$ (vs. $\mathrm{Li} / \mathrm{Li}^{+}$), a fivemembered cyclic ammonium (pyrrolidium) is selected as a cation candidate rather than the commonly used imidazolium to avoid an electrochemical decomposition reaction (Fig. 2a) [28,29]. Their counter anion part is derivatized by variation in the halogen elements. Because the solubility of the IL is mainly governed by anion characteristics, such as electronic and steric effects, differentiation in the

(a)


Fig. 2. (a) The synthesis of ILs and (b) LSV results for standard electrolyte (black), 5\% IL-Cl (red), 5\% IL-Br (blue), and 5\% IL-I (green). 
anion structure seems to be beneficial for estimating the effect of the anion structure on the overall solubility of the IL. Based on this strategy, the target ILs were synthesized by a quaternization reaction and their chemical structure was confirmed by ${ }^{1} \mathrm{H}-\mathrm{NMR}$ spectroscopy. The electrochemical stability of the obtained ILs was analyzed by linear sweep voltammetry (LSV) to confirm the electrochemical windows (Fig. 2b). Although irreversible oxidation currents associated with anion decomposition are observed at $3.1 \mathrm{~V}$ (vs. $\mathrm{Li} / \mathrm{Li}^{+}$for IL-I), $3.5 \mathrm{~V}$ (vs. Li/ $\mathrm{Li}^{+}$for IL-Br) and $3.9 \mathrm{~V}$ (vs. $\mathrm{Li} / \mathrm{Li}^{+}$for IL-Cl), they are still applicable because the ILs are quite stable under the common operating potential ranges for the Li-S cell.

To clarify whether the ILs promote dissolution of $l$ PS into the electrolyte, simple storage tests with the shortest polysulfide $\left(\mathrm{Li}_{2} \mathrm{~S}\right)$ and electrolyte were performed, as presented in Fig. 3a. The $\mathrm{Li}_{2} \mathrm{~S}$ is insoluble in the standard electrolyte even after three days. By contrast, the appearance of the IL-containing electrolyte was quite different: the solutions were transparent liquids without any precipitates, unlike the standard electrolyte. These results confirmed that the ILs apparently facilitate the dissolution of $l$-PS as to provide extra storage spaces in the electrolyte, thereby leading to higher utilization of $l$-PS in the Li-S cell. The performance of the ILs in a real Li-S cell was confirmed by examining the electrolytes by cyclic voltammetry $(\mathrm{CV})$ and their profiles were analyzed by calculation of the comparative ratio of each reduction step, as shown in Fig. 3b. The theoretical ratio of the first reduction step (1, from $\mathrm{S}_{8}$ to $\mathrm{Li}_{2} \mathrm{~S}_{4}, 418.0 \mathrm{~mA} \mathrm{~h}$ $\left.\mathrm{g}^{-1}\right)$ to the second reduction step $\left(2\right.$, from $\mathrm{Li}_{2} \mathrm{~S}_{4}$ to $\mathrm{Li}_{2} \mathrm{~S}, 1254 \mathrm{~mA} \mathrm{~h} \mathrm{~g}^{-1}$ ) is 3.00 , considering the electron transfer reaction at each electrochemical process. In practice, the standard electrolyte showed a value of 2.50 - the amount of the first step currents corresponded to an $\mathrm{S}_{8}$ conversion and was almost identical when compared to its analogues; however, their second electrochemical reaction seemed to be seriously disrupted, resulting in a decreasing ratio of 1 to 2 . This is a common behavior in the Li-S cell: the sluggish electrochemical reaction of solid-solid conversion at the second step accounts for the increasing internal resistance as $l$-PS accumulates on the cathode surface, which leads to continuous capacity fading of the Li-S cell as the electrochemical activity of the carbon cathode decreases. Conversely, the elec-
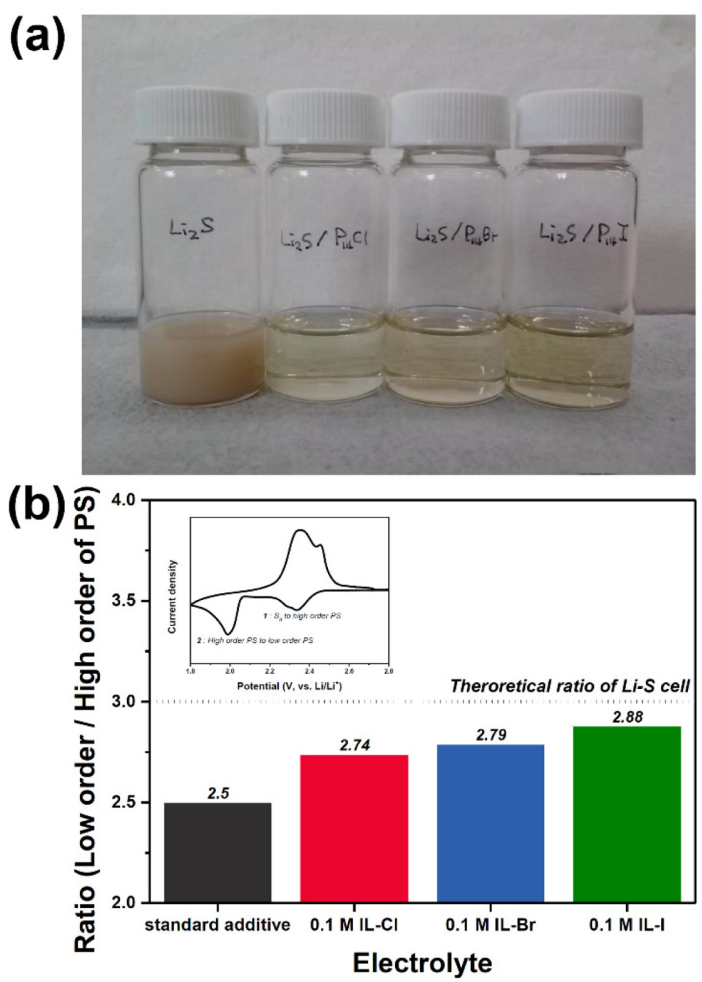

Fig. 3. (a) The results for dissolution test of $l$-PS and ILs and (b) the ratio of $h$-PS and $l$-PS and CV results for dissolution of PS (top left).

trolyte controlled with $0.1 \mathrm{M}$ ILs exhibited much higher reduction currents at the second step (ratio of 1/2: 2.74 for IL-Cl, 2.79 for IL-Br, and 2.88 for IL-I) as it accelerated the conversion reaction of $l$-PS. Note that these results are in good agreement with the previous analysis as shown in Fig. 2, where a higher solubility electrolyte $(\mathrm{I}>\mathrm{Br}>\mathrm{Cl})$ seems to be more effective at promoting the sluggish electrochemical reaction as the insulating $l$-PS is stored in the electrolyte instead of on the carbon cathode. These results confirmed the effectiveness of ILs in accelerating the electrochemical conversion reaction of $l$-PS in the Li$\mathrm{S}$ cell.

The cycle performances in response to the additive indicated an effect of the ILs on the electrochemical behavior of the Li-S cell (Fig. 4a). Because of their desirable solubility, the initial discharge capacity of the ILcontaining electrolyte was higher than that of the cell cycled with the standard electrolyte $\left(970.3 \mathrm{~mA} \mathrm{~h} \mathrm{~g}^{-1}\right.$ for IL-I, $764.5 \mathrm{~mA} \mathrm{~h} \mathrm{~g}^{-1}$ for IL-Br, $680.8 \mathrm{~mA} \mathrm{~h} \mathrm{~g}^{-1}$ for IL- 
(a)

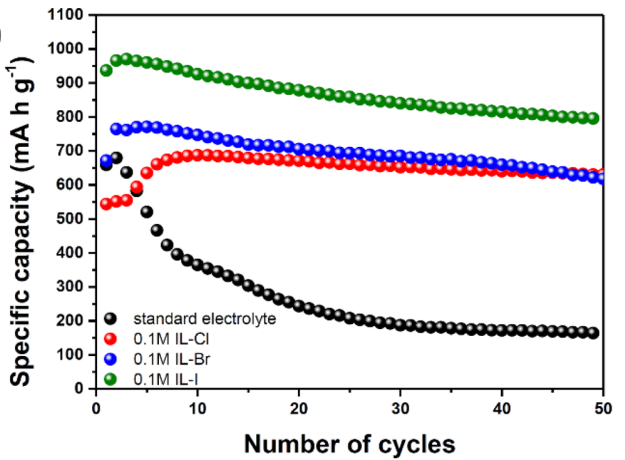

(b)

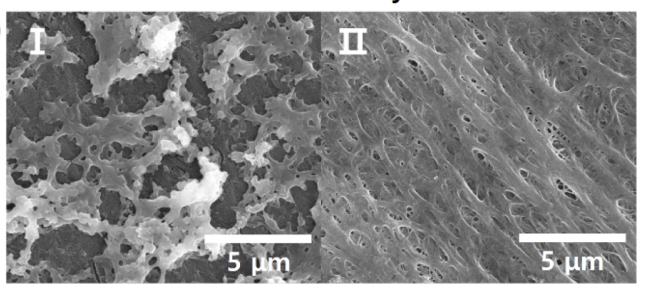

Fig. 4. (a) Electrochemical performances of coin cells cycled with standard electrolyte (black), electrolyte with IL-Cl added (red), IL-Br added (blue), and IL-I added (green) and (b) SEM images of PE separator cycled with standard electrolyte (I) and IL additives (II).

$\mathrm{Cl}$, and $636.9 \mathrm{~mA} \mathrm{~h} \mathrm{~g}^{-1}$ for the standard electrolyte). In addition, the IL-containing electrolyte showed a higher capacity retention, whereas the standard electrolyte exhibited a drastic capacity fading behavior as the number of cycles increased. Additional SEM analysis for cycled separators also indicate effectiveness of IL additives (Fig. 4b): pores in the PE separator cycled with only standard electrolyte were highly clogged while the cycled PE separator with IL additive still remained their porous structure as IL additive readily dissolved PS species during electrochemical charging/discharging processes. These results demonstrate that the ILs are effective at enhancing the electrochemical performance of the Li-S cell. The use of the ILs generated a better surface property of the cathode, resulting in a much higher specific capacity and better retention when compared to the non-controlled electrolyte.

\section{Conclusions}

In a conclusion, we have developed a novel approach to promote the slow electrochemical conversion of Li-S batteries. The slow kinetics of the Li-S cell mainly correspond to the isolation of $l$-PS on the cathode surface, which disrupted further electrochemical conversions. Using ILs as functional additives provided additional storage space in the electrolyte and facilitated further electron transfer reactions on the cathode surface. The chemical structure of the ILs was designed considering both the electrochemical stability and solubility with a variation in the anion structure, and pyrrolidinium iodide was determined to be the most effective IL in terms of the greater solubility of $l$-PS. The cycle performance of the cell with IL-I indicated excellent performance, as IL-I showed a remarkable initial capacity and a moderate capacity retention based on a higher utilization of $l$-PS in the Li-S cell. The results indicate that control of the interfacial chemistry between cathode/electrolyte is quite effective for improving the overall performance and this understanding of the role of IL will be useful for the construction of efficient Li-S batteries.

\section{Acknowledgement}

This work was supported by Incheon National University Research Grant in 2020.

\section{References}

[1] H. S. Kim, T. -G. Jeong, Y. -T. Kim, J. Electrochem. Sci. Technol., 2016, 7(3), 228-233.

[2] X. Zhao, G. Cheruvally, C. Kim, K. -K. Cho, H. -J. Ahn, K. -W. Kim, J. -H. Ahn, J. Electrochem. Sci. Technol., 2016, 7(2), 97-114.

[3] R. Yan, M. Oschatz, F. Wu, Carbon., 2020, 161, 162168.

[4] M. Rana, S. A. Ahad, M. Li, B. Luo, L. Wang, I. Gentle, R. Knibbe, Energy Storage Mater., 2019, 18, 289-310.

[5] L. Wang, Z. Y. Wang, J. F. Wu, G. R. Li, S. Liu, X. P. Gao, Nano Energy., 2020, 77, 105173.

[6] J. W. Park, J. Kang, J. Y. Koh, A. Caron, S. Kim, Y. Jung, J. Electrochem. Sci. Technol., 2020, 11(1), 33-40.

[7] M. Chen, X. Zhao, Y. Li, P. Zeng, H. Liu, H. Yu, M. Wu, Z. Li, D. Shao, C. Miao, G. Chen, H. Shu, Y. Pei, X. Wang, Chem. Eng. J., 2020, 385, 123905.

[8] J. L. Cheong, A. A. AbdelHamid, J. Y. Ying, Nano Energy., 2019, 66, 104114.

[9] T. Li, X. Bai, U. Gulzar, Y. -J. Bai, C. Capiglia, W. Deng, X. Zhou, Z. Liu, Z. Feng, R. P. Zaccaria, $A d v$. Funct. Mater., 2019, 29(32), 1901730.

[10] S. Lin, M. K. Shafique, Z. Cai, J. Xiao, Y. Chen, Y. Wang, X. Hu, ACS Nano., 2019, 13(11), 13037-13046. 
[11] S. Xiong, K. Xie, Y. Diao, X. Hong, Electrochim. Acta., 2012, 83, 78-86.

[12] J. K. Kang, T. J. Kim, J. W. Park, Y. Jung, The Bull. Korean Chem. Soc., 2019, 40(6), 566-571.

[13] T. Mukra, Y. Horowitz, I. Shekhtman, M. Goor, S. Drvarič Talian, L. Burstein, J. Kasnatscheew, P. Meister, M. Grünebaum, M. Winter, H. D. Wiemhöfer, D. Golodnitsky, E. Peled, Electrochim. Acta., 2019, 307, 76-82.

[14] J. Shim, T. J. Ko, K. Yoo, J. Ind. Eng. Chem., 2019, 80, 283-291.

[15] S. Duangdangchote, A. Krittayavathananon, N. Phattharasupakun, M. Sawangphruk, ECS Trans., 2020, 97(7), 827.

[16] S. S. Zhang, J. Power Sources., 2013, 231, 153-162.

[17] J. Xu, W. Zhang, H. Fan, F. Cheng, D. Su, G. Wang, Nano Energy., 2018, 51, 73-82.

[18] C. Y. Fan, Y. P. Zheng, X. H. Zhang, Y. H. Shi, S. Y. Liu, H. C. Wang, X. L. Wu, H. Z. Sun, J. P. Zhang, $A d v$. Energy Mater., 2018, 8(18), 1703638.

[19] Z. Yuan, H. -J. Peng, T. -Z. Hou, J. Q. Huang, C. -M. Chen, D. W. Wang, X. B. Cheng, F. Wei, Q. Zhang,
Nano Lett., 2016, 16(1), 519-527.

[20] Z. -L. Xu, J. -K. Kim, K. Kang, Nano Today., 2018, 19, 84-107.

[21] M. Rana, B. Luo, M. R. Kaiser, I. Gentle, R. Knibbe, $J$. Energy Chem., 2020, 42, 195-209.

[22] S. Menne, R. S. Kühnel, A. Balducci, Electrochim. Acta., 2013, 90, 641-648.

[23] S. Wilken, S. Xiong, J. Scheers, P. Jacobsson, P. Johansson, J. Power Sources., 2015, 275, 935-942.

[24] N. Plylahan, M. Kerner, D. -H. Lim, A. Matic, P. Johansson, Electrochim. Acta., 2016, 216, 24-34.

[25] S. Kim, Y. Jung, S. J. Park, Electrochim. Acta., 2007, 52(6), 2116-2122.

[26] J. H. Shin, E. J. Cairns, J. Power Sources., 2008, 177(2), 537-545.

[27] Y. Yan, J. -C. Xie, Y. Zhao, Y. Zhang, N. Cui, C. Li, C. Hao, J. Alloys Compd., 2019, 805, 733-739.

[28] S. Asha, K. P. Vijayalakshmi, B. K. George, Int. J. Quantum Chem., 2019, 119(22), e26014.

[29] W. Zhang, Y. Wang, X. Lan, Y. Huo, Res. Chem. Intermed., 2020, 46(6), 3007-3023. 